\title{
Presence of Resistant DEC Strains in a Tertiary Healthcare Center in North East India in Children under 18 Years
}

\author{
Abhijit Kumar Prasad ${ }^{10}$ \\ Wihiwot Valarie Lyngdoh ${ }^{1}$ \\ ${ }^{1}$ Department of Microbiology, North Eastern Indira Gandhi Regional \\ Institute of Health and Medical Sciences (NEIGRIHMS), Shillong, \\ Meghalaya, India \\ ${ }^{2}$ Manipur Health Department, Community Health Centre, Imphal, \\ Manipur, India \\ ${ }^{3}$ Department of Microbiology, ICMR-NIIRNCD, Jodhpur, Rajasthan, \\ India
}

Thigujam Surbala Devi ${ }^{2}$

Elantamilan Durairaj ${ }^{3(0)}$

Address for correspondence Wihiwot Valarie Lyngdoh, MD, Department of Microbiology, NEIGRIHMS, Shillong, 793018 Meghalaya, India (e-mail: drvalarielyngdoh@gmail.com).

J Lab Physicians 2022;14:278-283.

\begin{abstract}
Introduction Diarrheal illness such as diarrheagenic Escherichia coli (DEC), apart from rotavirus, is a common etiological agent known to cause moderate-to-severe diarrhea in low-income countries where unregulated use of antibiotics is rampant, giving rise to multidrug resistant (MDR) strains. This study is an earnest effort in reflecting the resistance pattern in such isolates.

Materials and Methods It is a hospital-based cross-sectional study conducted over a period of 1 year (January to December, 2015). Children aged less than 18 years presenting with $(n=170)$ and without $(n=47)$ diarrhea were included as cases and controls, respectively. Fresh stool sample from eligible participants was collected and inoculated on MacConkey agar. Based on the colony morphology and biochemical identification followed by polymerase chain reaction (PCR), different pathotypes of DEC were identified. All such isolates were subjected to antimicrobial susceptibility testing employing VITEK 2 identification system. The result of the tested antibiotics was evaluated as per Clinical and Laboratory Standards Institute 2015 guidelines.

Results DEC with specific virulence genes were detected by multiplex real-time PCR in 39

\section{Keywords}

- children under 18 years

- DEC

- diarrheagenic Escherichia coli

- MDR strains

- MDR-DEC

- North East India

- resistant DEC and 3 children with or without diarrhea, respectively. Most common DEC pathotypes found were enteroaggregative $E$. coli (38\%) followed by enteropathogenic $E$. coli (28.5\%). MDR isolates comprised 35 of $42 \mathrm{DEC}$ pathotypes (83.3\%). Resistance among DEC pathotypes to ampicillin, amoxicillin-clavulanate, ciprofloxacin, cephalosporin, nalidixic acid, imipenem, and cotrimoxazole was found to be statistically significant in comparison to non-DEC isolates. Conclusion This study has highlighted the increased prevalence of MDR strains among DEC pathotypes. Looking for these isolates will help detect dreadful DEC pathotypes like enterohemorrhagic E. coli where early administration of a sensitive antibiotic will go a long way in preventing complication like hemorrhagic colitis and hemolytic uremic syndrome.
\end{abstract}

published online February 9, 2022
DOI https://doi.org/ $10.1055 / \mathrm{s}-0042-1742421$. ISSN $0974-2727$. (c) 2022. The Indian Association of Laboratory Physicians. All rights reserved.

This is an open access article published by Thieme under the terms of the Creative Commons Attribution-NonDerivative-NonCommercial-License, permitting copying and reproduction so long as the original work is given appropriate credit. Contents may not be used for commercial purposes, or adapted, remixed, transformed or built upon. (https://creativecommons.org/ licenses/by-nc-nd/4.0/)

Thieme Medical and Scientific Publishers Pvt. Ltd., A-12, 2nd Floor, Sector 2, Noida-201301 UP, India 


\section{Introduction}

Diarrheal diseases are a leading cause of death in children under 5 years of age. Globally, there are nearly 1.7 billion cases of childhood diarrheal disease every year of which 525,000 succumb to it. ${ }^{1}$ Diarrhea accounts for 1 and $10 \%$ of deaths in neonates and children from 1 to 4 years, respectively. ${ }^{2}$ The integrated Global Action Plan for the Prevention and Control of Pneumonia and Diarrhea proposes a cohesive approach to ending preventable pneumonia and diarrhea deaths. The goal is ambitious but achievable to end preventable childhood deaths due to pneumonia and diarrhea by $2025 .^{2}$ They are both preventable and treatable. Rotavirus and Escherichia coli are the two most common etiological agents of moderate-to-severe diarrhea in low-income countries. E. coli are gram-negative bacteria that inhabit the gastrointestinal tract. Most strains do not cause illness. However, there are certain strains that may cause diarrhea and are categorized into various pathotypes on the basis of their virulence genes. Six pathotypes are associated with diarrhea (diarrheagenic): enterotoxigenic E. coli (ETEC), Shiga toxin-producing E. coli (STEC), enteropathogenic E. coli (EPEC), enteroaggregative $E$. coli (EAEC), enteroinvasive $E$. coli (EIEC), and possibly diffusely adherent $E$. coli (DAEC).

Interventions to prevent diarrhea, including safe drinking-water, use of improved sanitation, and hand washing with soap, can reduce disease risk. Diarrhea should be treated with oral rehydration solution, a solution of clean water, sugar, and salt. In addition, a 10 to 14-day supplemental treatment course of dispersible 10 to $20 \mathrm{mg}$ zinc tablets shortens diarrhea duration and improves outcomes. ${ }^{3}$ Antimicrobials are reliably helpful only for children with bloody diarrhea (probable shigellosis), suspected cholera with severe dehydration, and serious nonintestinal infections such as pneumonia. Antiprotozoal drugs are rarely indicated. $^{3}$

Antimicrobials should not be used routinely as it is not possible to distinguish clinical episodes that might respond, such as diarrhea caused by ETEC, from those caused by agents unresponsive to antimicrobials, such as rotavirus or Cryptosporidium. However, in developing countries like ours there is rampant unscrupulous usage of antimicrobials that has contributed immensely to the emergence and spread of multidrug resistant (MDR) strains of diarrheagenic E. coli (DEC). In view of this, there is considerable lack of understanding of the resistant pattern seen in the various strains of diarrheagenic $E$. coli. There are very few studies that have investigated the prevalence of diarrheagenic E. coli and their resistant pattern in the country generally and in the North East (NE) India in particular. Although there are sporadic studies, they are very few to formulate national guidelines on the management of acute diarrheal diseases reflecting the resistant pattern among diarrheagenic E. coli. This study was undertaken to investigate the prevalence and drug resistance pattern exhibited by the various pathotypes of diarrheagenic E. coli.

\section{Materials and Methods}

\section{Study Design}

This was a hospital-based cross-sectional study conducted in the department of microbiology of a tertiary care center over a period of 1 year (January to December, 2015). Here, the study participants were divided into two groups: one having diarrhea for any duration, while other not having diarrhea for the last 1 month. The prevalence of DEC strains in both groups was noted and antimicrobial susceptibility test was conducted for all of them.

\section{Inclusion Criteria for Study Participants}

All pediatric patients ( $<18$ years of age) with acute diarrhea that was defined as an increase in fluidity, volume, and number of stools passed relative to usual bowel habits of each individual within 24 hour and lasting not longer than 14 days were enrolled in the study. Fever was defined as a temperature of greater than or equal to $37.5^{\circ} \mathrm{C}$. If the parents or legal guardians accepted participation in the study, patients with acute diarrhea attending the outpatient and inpatient department of pediatrics were enrolled in this study. The isolates that were resistant to 2 or more groups of drugs were labeled as MDR isolates.

\section{Control}

Children ( $<18$ years of age) with no history of diarrhea for at least 1 month were included as controls.

\section{Exclusion Criteria}

Children with diarrhea that was attributed to classic pathogens such as Salmonella spp./Shigella spp./Vibrio spp. or gross infestation with parasites were excluded from the study. In addition, either cases or controls treated with antibiotics 1 week before the collection of stool samples were excluded.

\section{Ethical Approval}

Ethical approval was obtained from the institution ethics committee.

\section{Sample Collection}

Stool samples were obtained from 170 children with diarrhea (cases) and 47 from children without diarrhea (controls) and further processed and analyzed for the detection of DEC pathotypes as follows.

\section{Morphological and Biochemical Identification of Escherichia coli}

Fresh stool sample from the participants was inoculated and streaked onto the surface of MacConkey agar (HiMedia Laboratories Pvt. Ltd, Mumbai, India) for isolated colonies. Characteristic discrete lactose fermenting colonies produced after 24 hours of incubation aerobically at $37^{\circ} \mathrm{C}$ were streaked onto fresh sterilized nutrient agar (HiMedia Laboratories Pvt. Ltd, Mumbai, India) and identified by conventional biochemical tests such as indole, ${ }^{4}$ methyl red, ${ }^{5}$ VogesProskauer, ${ }^{5}$ citrate, $^{6}$ and urease ${ }^{7}$ tests. The procedure of inoculation of isolates and interpretation of biochemicals 
were done as per protocols described by American Society of Microbiologists. ${ }^{4-7}$ Isolates that were positive to indole and methyl red tests but negative to Voges-Proskauer, citrate, and urease tests were identified as E. coli.

\section{Maintenance of Isolates}

Biochemically confirmed $E$. coli isolated from the stool samples was maintained in trypticase soy broth supplemented with 20\% glycerol (HiMedia Laboratories Pvt. Ltd, Mumbai, India) and nutrient agar slants (HiMedia Laboratories Pvt. Ltd, Mumbai, India) for the investigation of the genes encoding pathogenicity by molecular test.

\section{Molecular Analysis for Screening Diarrheagenic Escherichia coli Virulent Genes}

DNA was extracted from an overnight pure culture of $E$. coli using QIAamp DNA Mini Kit (Qiagen India Pvt. Ltd, New Delhi, India). The extracted DNA was subjected to multiplex real-time polymerase chain reaction (PCR) with high-resolution melting technology employing primer nucleotide sequences specific to target virulent genes of different DEC pathotypes that was designed by Sigma Aldrich, Bengaluru, based on the previously published sequences. ${ }^{8,9}$ Multiplex real-time PCR was performed using Rotor-Gene Q instrument (Qiagen) having high-resolution melt analyzer. Molecular analysis for screening diarrheagenic E. coli virulent genes formed the first part of this study that has been already been published in Indian Journal of Medical Microbiology in its Oct-Dec 2018 issue. $^{10}$

\section{Antibiotic Susceptibility Testing by Automated Method}

Antibiotic susceptibility testing to 18 antimicrobial agents including ampicillin, amoxicillin clavulanic acid, nalidixic acid, ciprofloxacin, trimethoprim-sulfamethoxazole, gentamicin, amikacin, ceftriaxone, cefuroxime, cefotaxime, cefuroxime axetil, imipenem, meropenem, piperacillintazobactam, tigecycline, colistin, ertapenem was determined for all isolated E. coli (DEC and non-DEC strains by using antimicrobial susceptibility AST-N280 card (bio-
MerieuxInc., France) in VITEK 2 identification System (VITEK 2 version 07.01, bioMerieux Inc., France). All the processing was done as per the antimicrobial susceptibility test (AST) card manual provided. The antibiotic susceptibility pattern and minimum inhibitory concentration (that is, lowest concentration of antimicrobial with no visible bacterial growth) of the $E$. coli isolates were evaluated and interpreted in accordance with Clinical and Laboratory Standards Institute 2015 guideline. The isolates that were resistant to 2 or more groups of drug were labeled as MDR isolates.

\section{Statistical Interpretation}

The data were collected and recorded using MS-Excel for Windows v2013. Summary statistics and analysis of significance were done using MedCalc v12.5.0 for Windows (MedCalc Software, Ostend, Belgium). The comparison of single and two proportions was done using chi-squared test and Fisher's exact test as applicable. The threshold for significance was considered at $p$-value less than 0.05 .

\section{Results}

The first part of the study was conducted to investigate the prevalence of DEC among the pediatric age group ( $<18$ years) presenting at the tertiary healthcare hospital with and without diarrhea, the findings of which has been published earlier in a reputed journal. ${ }^{10}$

During the study period of 1 year (January 2015 to December 2015), 170 children with diarrhea (cases) and 47 children without diarrhea (controls) were included in this study. A total of 217 nonduplicated biochemically confirmed $E$. coli isolates obtained from the stool samples of these children. Diarrheagenic E. coli with specific virulence genes were detected by multiplex real-time PCR in 39 of 170 children with diarrhea. However, only 3 of 47 children without diarrhea were found to harbor DEC-specific virulent genes. The break-up of the DEC isolates is depicted in - Table 1. As evident, neither cases nor controls harbored

Table 1 Diarrheagenic Escherichia coli with specific virulence genes detected by multiplex real-time PCR

\begin{tabular}{|l|l|l|l|l|l|}
\hline \multirow{2}{*}{$\begin{array}{l}\text { Diarrheagenic } \\
\text { E. coli pathotypes }\end{array}$} & \multirow{2}{*}{$\begin{array}{l}\text { Virulent genes } \\
\text { screened }\end{array}$} & \multicolumn{2}{|l|}{$\begin{array}{l}\text { Total no. of DEC isolates with specific } \\
\text { virulence gene among children }\end{array}$} & $\begin{array}{l}\text { Total DEC isolate } \\
(\boldsymbol{n}=\mathbf{4 2})\end{array}$ & MDR Isolates \\
\cline { 3 - 5 } & & $\begin{array}{l}\text { With diarrhea } \\
\boldsymbol{n}=\mathbf{1 7 0}(\%)\end{array}$ & $\begin{array}{l}\text { Without diarrhea/ } \\
\text { control group } \\
\boldsymbol{n}=\mathbf{4 7}(\%)\end{array}$ & \\
\hline EAEC & CVD 432 & $15(8.82)$ & $1(2.13)$ & $16(38 \%)$ & $11(68.75 \%)$ \\
\hline EIEC & ial & $2(1.18)$ & 0 & $2(4.7 \%)$ & 0 \\
\hline Atypical EPEC & eae & $10(5.88)$ & $2(4.26)$ & $12(28.5 \%)$ & $12(100 \%)$ \\
\hline Typical EPEC & eae \&bfp & $7(4.12)$ & 0 & $7(16.6 \%)$ & $7(100 \%)$ \\
\hline ETEC & stla & $5(2.94)$ & 0 & $5(11.9 \%)$ & $5(100 \%)$ \\
\hline Total & & $39(22.94)$ & $3(6.39)$ & 42 & $35(83.3 \%)$ \\
\hline
\end{tabular}

Abbreviations: DEC, diarrheagenic Escherichia coli; EAEC, enteroaggregative E. coli; EIEC, enteroinvasive E. coli; EPEC, enteropathogenic E. coli; ETEC, enterotoxigenic $E$. coli; MDR, multidrug resistant; $P C R$, polymerase chain reaction. 
Table 2 AST profile of DEC and non-DEC isolates

\begin{tabular}{|l|l|l|l|}
\hline Antimicrobial agent & $\begin{array}{l}\text { DEC } \\
(\boldsymbol{n}=39)\end{array}$ & $\begin{array}{l}\text { Non-DEC } \\
(\boldsymbol{n}=\mathbf{1 4 1})\end{array}$ & $p$-Value \\
\cline { 2 - 4 } & Res \% & Res \% & \\
\hline Ampicillin & 84.21 & 62.5 & $p=0.0260^{\mathrm{a}}$ \\
\hline $\begin{array}{l}\text { Amoxicillin/ } \\
\text { clavulanic acid }\end{array}$ & 42.11 & 20 & $p<0.0001^{\mathrm{a}}$ \\
\hline Amikacin & 10.53 & 5 & $p=0.3231^{\mathrm{b}}$ \\
\hline Ciprofloxacin & 68.42 & 35 & $p=0.0002^{\mathrm{a}}$ \\
\hline Ceftriaxone & 52.63 & 30 & $p=0.0013^{\mathrm{a}}$ \\
\hline Cefuroxime & 63.16 & 30 & $p=0.0005^{\mathrm{a}}$ \\
\hline Cefuroxime axetil & 63.16 & 30 & $p=0.0005^{\mathrm{a}}$ \\
\hline Ertapenem & 5.26 & 5 & $p=0.7465^{\mathrm{b}}$ \\
\hline Cefepime & 21.05 & 15 & $p=0.011^{\mathrm{a}}$ \\
\hline Gentamicin & 15.79 & 15 & $p=0.9113^{\mathrm{b}}$ \\
\hline Imipenem & 5.26 & 0 & $p=0.0283^{\mathrm{a}}$ \\
\hline Meropenem & 5.26 & 2.5 & $p=0.6631^{\mathrm{b}}$ \\
\hline Nalidixic acid & 94.74 & 67.5 & $p=0.0008^{\mathrm{a}}$ \\
\hline $\begin{array}{l}\text { Cefoperazone/ } \\
\text { sulbactam }\end{array}$ & 5.26 & 10 & $p=0.855^{\mathrm{b}}$ \\
\hline $\begin{array}{l}\text { Trimethoprim/ } \\
\text { sulfamethoxazole }\end{array}$ & 94.74 & 52.5 & $p<0.0001^{\mathrm{a}}$ \\
\hline $\begin{array}{l}\text { Piperacillin/ } \\
\text { tazobactam }\end{array}$ & 15.79 & 10 & $p=0.4245^{\mathrm{b}}$ \\
\hline
\end{tabular}

Abbreviations: AST, aspartate aminotransferase; DEC, diarrheagenic Escherichia coli; Res, resistance.

${ }^{a}$ Significant difference exists between DEC and non-DEC.

${ }^{b}$ No significant difference between DEC and non-DEC isolates.

genes for enterohemorrhagic E. coli (EHEC). These results agree with the low prevalence of EHEC infection in developing countries The AST profile of DEC and non-DEC isolates is illustrated in - Table 2. The antimicrobial resistant profile among DEC pathotype is shown in - Table 3. EIEC was isolated from two patients with diarrhea. However, both were sensitive to all the panel of antibiotics put up. All DEC isolates were found to be sensitive to colistin and tigecycline (not reflected in the table).

\section{Discussion}

E. coli is identified as an important cause of pediatric diarrhea in developing countries. Although DEC pathotypes are well recognized, they are not routinely sought due to lack of infrastructures such as antisera and advanced molecular techniques. Thus, the exact burden of $E$. coli diarrhea among the hospitalized children across India especially Northeast India is still unclear. There are few studies from Mizoram, a state located in North East India in adjoining Myanmar and Bangladesh where limited study has been conducted on the prevalence of diarrhea associated with DEC..11,12 There is another in-depth study conducted by Chellapandi et al on the prevalence of MDR-DEC pathotypes isolated from children with or without diarrhea in North Indian population. ${ }^{13}$ In our study, there was significant association of DEC with diarrhea group in comparison to nondiarrhea group $(p=0.0195)$ as is seen in other studies. ${ }^{13}$ This finding is good evidence that DEC plays an important role in the development of diarrhea in children. However, the isolation of DEC pathotypes from nondiarrhea group suggests that healthy children may act as their carrier. However, a study on a larger sample size may be needed to further strengthen this finding.

The incidence of diarrhea due to MDR E. coli has increased (50-70\%) in developing countries and up to $75 \%$ has been reported from India. ${ }^{14}$ In our study, there was significant difference in the antimicrobial resistance pattern observed among the DEC isolates relative to non-DEC isolates for antibiotics like ampicillin, trimethoprim sulfamethoxazole, nalidixic acid, ciprofloxacin, ceftriaxone, cefuroxime, cefepime, amoxicillin-clavulanic acid, and imipenem. DEC isolates were more resistant to most of the antibiotics tested. Our findings were in concordance with a study performed by Sudershan et al in 2014 which concluded that most of the E. coli isolates from children with diarrhea were resistant to norfloxacin, amoxycillin, co-trimoxazole, ampicillin, ceftriaxone, cefotaxime, and metronidazole. ${ }^{15} \mathrm{~A}$ similar study done by Alikhani et al in Iran also supported the antibiotic resistance pattern observed in our study. ${ }^{14}$ In our study, 35 (83.3\%) DEC isolates were found to be MDR that was much higher than findings published by Chellapandi et al (41.4\%). ${ }^{13}$ This gross difference may be due to varying perception of MDR definition. In our case, resistance to 2 or more groups of antibiotics was considered as MDR as opposed to other study, where resistance to 3 to 5 groups of antibiotics may be considered MDR.

EPEC and ETEC isolates were found to show high level of resistance to most generic drugs used in the study namely penicillin, fluoroquinolones, cotrimoxazole, and second- and third-generation cephalosporins, while low level of resistance to aminoglycosides, fourth generation cephalosporin, penicillin and $\beta$-lactamase combination, and carbapenems. This was in concordance with studies conducted elsewhere in the country. ${ }^{14,16}$ A study by Ochoa et al found their DEC isolate to be more sensitive to cephalosporin as opposed to our study. ${ }^{17}$

There were only two patients with diarrhea from whom EIEC was isolated. However, both were sensitive to all the panel of antibiotics put up that was discordant to studies from other parts of the country. ${ }^{13}$ In India, very few reports are available on the occurrence of EIEC associated with children diarrhea. ${ }^{13,16}$ As evident in many studies, the isolation of EIEC isolate among DEC pathotype is very few or none which explains the increased susceptibility of these isolates to antimicrobials. ${ }^{14,17}$ Due to its low prevalence, its exposure to antimicrobials has been low and hence its resistance developing mechanism has not evolved. However, antimicrobial testing on increased number of EIEC isolates will be needed to corroborate our findings.

\section{Conclusion}

This study has highlighted the presence of MDR-DEC isolates in children population of the North East region of country. It 
Table 3 Antimicrobial resistance profile among DEC pathotypes

\begin{tabular}{|l|l|l|l|l|}
\hline \multirow{2}{*}{ Antibiotic } & Resistance & \multicolumn{2}{l|}{} \\
\cline { 2 - 5 } & EPEC $(\boldsymbol{n}=\mathbf{1 7})$ & $\begin{array}{l}\text { ETEC } \\
(\boldsymbol{n}=\mathbf{5})\end{array}$ & $\begin{array}{l}\text { EAEC } \\
(\boldsymbol{n}=\mathbf{1 5})\end{array}$ & $\begin{array}{l}\text { DEC } \\
\text { (Total) }\end{array}$ \\
\hline Ampicillin & $17(100)$ & $5(100)$ & $12(75)$ & 84.21 \\
\hline Amoxicillin/clavulanic acid & $10(58.8)$ & $2(40)$ & $6(37.5)$ & 42.11 \\
\hline Amikacin & $3(17.6)$ & 0 & $1(6.25)$ & 10.53 \\
\hline Ciprofloxacin & 100 & $3(60)$ & $9(56.2)$ & 68.42 \\
\hline Ceftriaxone & $10(58.8)$ & $5(100)$ & $6(37.5)$ & 52.63 \\
\hline Colistin & 0 & 0 & 0 & 0 \\
\hline Cefuroxime & $14(82.3)$ & $5(100)$ & $7(43.7)$ & 63.16 \\
\hline Cefuroxime axetil & $14(82.3)$ & $5(100)$ & $7(43.7)$ & 63.16 \\
\hline Ertapenem & 0 & 0 & $1(6.25)$ & 5.26 \\
\hline Cefepime & $3(17.6)$ & $3(60)$ & $1(6.25)$ & 21.05 \\
\hline Gentamicin & $7(41.1)$ & 0 & $1(6.25)$ & 15.79 \\
\hline Imipenem & 0 & 0 & $1(6.25)$ & 5.26 \\
\hline Meropenem & 0 & 0 & $1(6.25)$ & 5.26 \\
\hline Nalidixic acid & $17(100)$ & $5(100)$ & $14(87.5)$ & 94.74 \\
\hline Cefoperazone/sulbactam & 0 & 0 & $1(6.25)$ & 5.26 \\
\hline Trimethoprim/sulfamethoxazole & $17(100)$ & $5(100)$ & $14(87.5)$ & 94.74 \\
\hline Tigecycline & 0 & 0 & 0 & 0 \\
\hline Piperacillin/tazobactam & $3(17.6)$ & $2(40)$ & $1(6.25)$ & 15.79 \\
\hline
\end{tabular}

Abbreviations: DEC, diarrheagenic Escherichia coli; EAEC, enteroaggregative E. coli; EPEC, enteropathogenic E. coli; ETEC, enterotoxigenic E. coli. Note: \% mentioned in parenthesis.

has reiterated the importance of looking for DEC pathotypes in children presenting with diarrhea before starting any antibiotic as viral enteropathogens are common cause of diarrhea in children. This will help to reduce abuse of antibiotics that in turn will reduce emergence of MDR strains. DEC isolates whenever isolated should be subjected to antimicrobial susceptibility testing so that appropriate antibiotics guided by AST results may be administered if indicated. These indications may include dysentery, severe or prolonged disease, eradication of fecal shedding, and transmission and prevention of sequelae and death. ${ }^{18}$ It is imperative to implement strategies to prevent and control the emergence and spread of resistant organisms by improving diagnosis by way of molecular testing or use of easy and rapid test such as E. coli O-specific antisera to screen for DEC strains and reducing the selective pressure caused by overuse and misuse of antibiotics in children.

\section{Funding}

None.

\section{Conflicts of Interest}

None declared.

\section{Acknowledgments}

The authors would like to take this opportunity to thank our laboratory technician Jedidah Lungleng, Sony Gandhi, and Kevizenou Miari who were of enormous help in collection and processing of samples from study subjects.

\section{References}

1 World Health Organization. Diarrhoeal Diseases. 2017. Accessed January 7, 2021: https://www.who.int/news-room/fact-sheets/detail/diarrhoeal-disease

2 World Health Organization UNICEF. Ending preventable child deaths from pneumonia and diarrhoea by 2025: the integrated Global Action Plan for Pneumonia and Diarrhoea (GAPPD). 2013. Accessed September 24, 2020. Accessed January 7, 2021: http:// www.defeatdd.org/sites/default/files/node-images/gappd-fullreport.pdf

3 World Health Organization. First steps for managing an outbreak of acute diarrhoea. 2010. Last accessed on January 17, 2022, at: https://apps.who.int/iris/rest/bitstreams/66796/retrieve

4 MacWilliams MP. Indole Test Protocols. American Society of Microbiology. 2009. Accessed January 7, 2021: https://asm.org/Protocols/Indole-Test-Protocol

5 McDevitt S. Methyl Red and Voges-Proskauer Test Protocols. American Society for Microbiology 2009. Accessed January 7, 2021 https://asm.org/Protocols/Methyl-Red-and-Voges-Proskauer-Test-Protocols

6 MacWilliams MPCitrate Test Protocols. American Society of Microbiology. 2009. Accessed January 7, 2021: https://asm.org/Protocols/Citrate-Test-Protocol

7 Brink BUrease Test Protocols. American Society of Microbiology. 2011. Accessed January 7, 2021: https://asm.org/Protocols/Urease-Test

8 Aranda KRS, Fagundes-Neto U, Scaletsky ICA. Evaluation of multiplex PCRs for diagnosis of infection with diarrheagenic 
Escherichia coli and Shigella spp. J Clin Microbiol 2004;42(12): 5849-5853

9 Brandal LT, Lindstedt B-A, Aas L, Stavnes TL, Lassen J, Kapperud G. Octaplex PCR and fluorescence-based capillary electrophoresis for identification of human diarrheagenic Escherichia coli and Shigella spp. J Microbiol Methods 2007;68(02):331-341

10 Devi TS, Durairaj E, Lyngdoh WV, Duwarah SG, Khyriem AB, Lyngdoh CJ. Real-time multiplex polymerase chain reaction with high-resolution melting-curve analysis for the diagnosis of enteric infections associated with diarrheagenic Escherichia coli. Indian J Med Microbiol 2018;36(04):547-556

11 Dutta TK, Warjri I, Roychoudhury P, et al. Extended-spectrum- $\beta$ lactamase-producing Escherichia coli isolate possessing the Shiga toxin gene (stx1) belonging to the 064 serogroup associated with human disease in India. J Clin Microbiol 2013;51(06):2008-2009

12 Begum J, Dutta TK, Chandra R, et al. Molecular and phenotypic characterization of shigatoxigenic Escherichia coli (STEC) and enteropathogenic E. coli (EPEC) from piglets and infants associated with diarrhoea in Mizoram, India. Afr J Biotechnol 2014; $\cdots: 13$
13 Chellapandi K, Dutta TK, Sharma I, De Mandal S, Kumar NS, Ralte L. Prevalence of multi drug resistant enteropathogenic and enteroinvasive Escherichia coli isolated from children with and without diarrhea in Northeast Indian population. Ann Clin Microbiol Antimicrob 2017;16(01):49

14 Alikhani MY, Hashemi SH, Aslani MM, Farajnia S. Prevalence and antibiotic resistance patterns of diarrheagenic Escherichia coli isolated from adolescents and adults in Hamedan, Western Iran. Iran J Microbiol 2013;5(01):42-47

15 Sudershan RV, Kumar RN, Kulkarni B, et al. E. coli pathotypes and their antibiotic resistance in young children with diarrhea in Hyderabad, India. Int J Curr Microbiol Appl Sci 2014;3:647-654

16 Rajeshwari K, Beena U, Singh R, et al. Multi drug resistant enteropathogenic E. coli diarrhea in children. Am J Res Commun 2015;3:27-48

17 Ochoa TJ, Ruiz J, Molina M, et al. High frequency of antimicrobial drug resistance of diarrheagenic Escherichia coli in infants in Peru. Am J Trop Med Hyg 2009;81(02):296-301

18 Pickering LK. Antimicrobial resistance among enteric pathogens. Semin Pediatr Infect Dis 2004;15(02):71-77 